\title{
X-ray Computed Tomography Instrument Performance Evaluation, Part I: Sensitivity to Detector Geometry Errors
}

\author{
Bala Muralikrishnan, Meghan Shilling, Steve Phillips ${ }^{1}$, Wei Ren, Vincent Lee, and Felix Kim \\ National Institute of Standards and Technology, \\ Gaithersburg, MD 20899 USA \\ balam@nist.gov \\ katharine.shilling@nist.gov \\ wei.ren@nist.gov \\ vincent.d.lee@nist.gov \\ felix.kim@nist.gov
}

$\mathrm{X}$-ray computed tomography (XCT), long used in medical imaging and defect inspection, is now increasingly used for dimensional measurements of geometrical features in engineering components. With widespread use of XCT instruments, there is growing need for the development of standardized test procedures to verify manufacturer specifications and provide pathways to establish metrological traceability. As technical committees within the American Society of Mechanical Engineers (ASME) and the International Organization for Standardization (ISO) are developing documentary standards that include test procedures that are sensitive to all known error sources, we report on work exploring one set of error sources, instrument geometry errors, and their effect on dimensional measurements. In particular, we studied detector and rotation stage errors in cone-beam XCT instruments and determined their influence on sphere center-to-center distance errors and sphere form errors for spheres located in the tomographically reconstructed measurement volume. We developed a novel method, called the single-point ray tracing method, that allows for efficient determination of the sphere center-to-center distance error and sphere form error in the presence of each of the different geometry errors in an XCT instrument. In Part I of this work, we (1) describe the single-point ray tracing method, (2) discuss optimal placement of spheres so that sphere center-to-center distance errors and sphere form errors are sensitive to the different detector geometry errors, and (3) present data validating our method against the more conventional radiograph-based tomographic reconstruction method. In Part II of this work, we discuss optimal placement of spheres so that sphere center-to-center distance errors and sphere form errors are sensitive to error sources associated with the rotation stage. This work is in support of ongoing standards development activity within ASME and ISO for XCT performance evaluation.

Key words: cone-beam; distance error; documentary standards; form error; geometry errors; performance evaluation; radiographbased method; sensitivity analysis; single-point ray tracing method; X-ray computed tomography.

Accepted: April 24, 2019

Published: July 11, 2019

https://doi.org/10.6028/jres.124.014

\section{Introduction}

Motivated by the need to inspect parts with internal features, X-ray computed tomography (XCT) is transitioning from mainly defect inspection to also include dimensional metrology [1,2]. Despite the increasing use of this technology for dimensional measurements, issues such as metrological traceability, measurement uncertainty, and performance evaluation of commercial XCT instruments remain partially unresolved.

\footnotetext{
${ }^{1}$ Deceased.
} 
Documentary standards describing carefully constructed test procedures that are sensitive to all known error sources represent one route to demonstrating metrological traceability of dimensional measurements [3]. Both the American Society of Mechanical Engineers (ASME) and the International Organization for Standardization (ISO) have developed such standards for spherical and Cartesian coordinate measuring instruments (the ASME B89.4 and the ISO 10360 series), in which the test procedures are sensitive to all known error sources, and the results from the test procedures may be used as documentary evidence to demonstrate metrological traceability per ASME B89.7.5 [4]. In the case of XCT instruments, there is currently one published guideline, the VDI/VDE 2617-13 [5], but test procedures described in that document are not exhaustive. Working groups within ASME and ISO are currently independently developing performance verification standards.

The task of developing a documentary standard for XCT instruments is exceedingly challenging because of the number and complexity of error sources. Error sources include those arising from the instrument (source, detector, rotation stage), the workpiece, interaction of the instrument with the workpiece, data processing, the environment, and the operator [1, 6-7]. Exploring all error sources is beyond the scope of this work. We consider one class of error source in detail here: instrument geometry errors for cone-beam XCT instruments.

Ferrucci et al. [8] performed an exhaustive literature review on geometry errors in XCT instruments. Kumar et al. [9] attempted to quantify the effect of XCT geometry errors on dimensional measurements. The method presented in Ref. [9] consists of measuring scale bars of different lengths at different orientations in the measurement volume in the presence of various instrument geometry errors. The use of scale bars allows the determination of the behavior of geometry errors at those specific positions and orientations. However, our approach allows us to determine the most sensitive locations at which to perform measurements to detect these errors, i.e., to obtain the largest possible sphere center-to-center distance errors and sphere form errors arising from a particular instrument geometry error source - an aspect not explored by Kumar et al. [9]. Ferrucci et al. [10] explored the effects of detector misalignment by considering the displacement of reconstructed sphere centers distributed in the measurement volume. The authors of Ref. [10] did not consider sphere center-to-center distances and therefore did not provide insight on suitable test positions for performance evaluation. Ametova et al. [11] developed a novel method to determine the influence of instrument geometry errors by comparing the boundaries of the image after forward projection in the presence of instrument geometry errors against those obtained for a perfect system. Their method is computationally efficient but, again, does not provide recommendations for performance testing. Aloisi et al. [12] experimentally determined the effect of detector tilt angle errors on sphere center-to-center distance errors for a vertically oriented scale bar. While their experiments quantified the errors for that specific scale bar orientation, their work did not identify the most sensitive positions of the scale bar for individual geometry error sources.

In this paper (Part I), we discuss a novel method that can be used to efficiently compute the effects of geometry error sources associated with the detector (location and orientation errors); we address geometry error sources associated with the rotation stage (location, radial, axial, wobble, and encoder scale errors) in Part II. In each part, for each geometry error source, we evaluated the effect on sphere center-to-center distance errors and form errors for spheres distributed in the measurement volume. This study was limited to the cone-beam configuration only and for the case of a stationary detector and nonhelical mode scanning.

Scale bars with spheres at the ends are commonly used in testing the performance of Cartesian and spherical coordinate measuring instruments. Also, as reported by Kumar et al. [9], scale bars are considered for performance testing of XCT instruments. A plate with spheres mounted in various locations is currently one reference object under consideration within ASME for performance testing of XCT instruments, and therefore we used spheres in this study. For our purposes in this paper, form error is considered to be the difference between the maximum and minimum residuals from a least-squares best-fit sphere fit to given point cloud data set associated with a spherical surface. 
We performed the distance and form error computations using two different methods. The first method is a radiograph-based method that takes several hours to execute, while the second is a novel single-point ray tracing method that is an approximate technique. The latter method takes less than $5 \mathrm{~min}$, yet it provides comparable results. One motivation for this work was to provide input to ongoing standards efforts within ASME and ISO on selecting appropriate sphere center locations that have high sensitivity (i.e., largest sphere center-to-center distance error and sphere form error) to the different geometry error sources in XCT instruments.

The rest of the paper is organized as follows. We describe the two methods in Sec. 2. We discuss geometry errors associated with the detector in Sec. 3. Discussion and conclusions follow in Sec. 4 and Sec. 5 , respectively. We discuss error sources associated with the rotation stage in Part II.

\section{Method}

\subsection{Coordinate System and Setup}

The geometric arrangement of the source, detector, and rotation axis is shown in Fig. 1 along with the coordinate system employed. The geometry errors considered in this paper and the coordinate system employed are based on Ferrucci et al. [13]. The origin of the global coordinate system is located at the source, $O$. The $Y$ axis is parallel to the rotation axis. A line is drawn from the source, orthogonal to the rotation axis, intersecting the rotation axis at point $P$, and intersecting the detector at point $D$. The $Z$ axis is coincident with this line, with the positive direction pointing away from the detector (as shown in Fig. 1). In an ideally aligned instrument, the $Z$ axis intersects the plane of the detector orthogonally at the detector's geometrical center. By definition, the $X$ and $Y$ coordinates of points $P$ and $D$ in the global coordinate system are zero. Point $P$ is also the center of the measurement volume. The location of point $D$ in the $U V$ detector coordinate system is given by $(u, v)$ and is assumed to be known from prior calibration. In an ideal instrument, the $U$ and $V$ axes of the detector coordinate system are respectively parallel to the $X$ and $Y$ axes of the global coordinate system. The origin of the detector coordinate system is located at the bottom-left corner of the detector, when viewing the detector from the source. The rotation stage index angle $\theta$ is zero on the positive $X$ axis and increases as shown in Fig. 1.

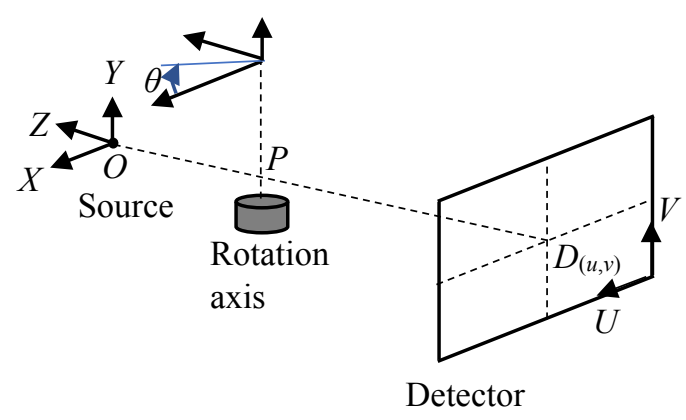

Fig. 1. Geometry of the instrument setup and coordinate system.

In the simulations described in Sec. 3 and Sec. 4, the rotation axis and the detector are located at distances of $400 \mathrm{~mm}$ and $1177 \mathrm{~mm}$ (based on Ref. [10]), respectively, along the $Z$ axis from the source. The detector is a two-dimensional array of $1501 \times 1501$ square pixels; the length of the pixel sides is $0.127 \mathrm{~mm}$. To capture the effect of geometry errors, we considered a reference object consisting of 125 spheres, each of nominal diameter $=3 \mathrm{~mm}$, equally distributed in 5 horizontal $(X Z)$ planes located at the following $Y$ positions: $-25 \mathrm{~mm},-12.5 \mathrm{~mm}, 0 \mathrm{~mm}, 12.5 \mathrm{~mm}$, and $25 \mathrm{~mm}$, as shown in Fig. 2(a). At each of these five levels, one sphere is located on the rotation axis, eight spheres are equally spaced on a circle of $25 \mathrm{~mm}$ diameter, and 16 spheres are equally spaced on a circle of $50 \mathrm{~mm}$ diameter (Fig. 2(b)). 


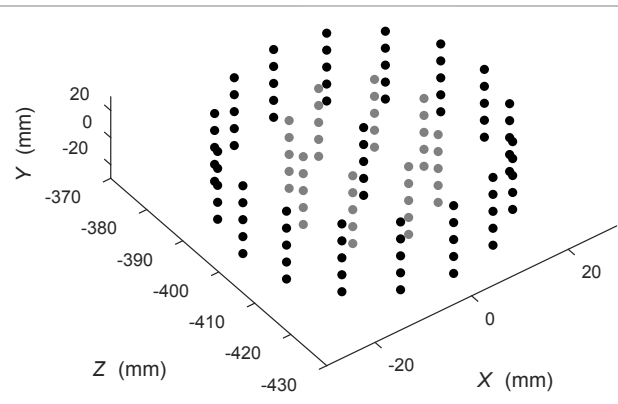

(a)

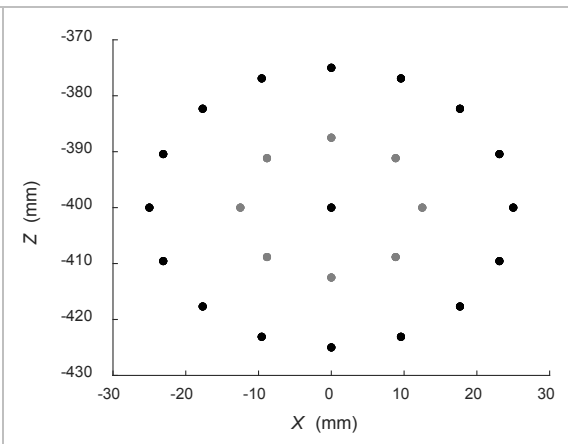

(b)

Fig. 2. (a) Three-dimensional view of the reference object consisting of 125 spheres. (b) Top view of the same object.

\subsection{Radiograph-Based Method}

The radiograph-based method is identical to a typical XCT workflow process with the exception that the radiographs are not experimentally acquired; rather, they are numerically generated. In the forwardprojection step of this method, sets of radiographs of the reference object are numerically generated in MATLAB (R2016b) ${ }^{2}$ in the presence of the imposed individual geometry error parameters considered in this paper. For each pixel in each radiograph, the attenuation is calculated analytically by constructing lines from the source to the center of each pixel and calculating the total intersection length of each constructed line with all spheres along its path. Radiographs are generated for 2400 equally spaced angular positions of the reference object over a full rotation of $360^{\circ}$.

In the back-projection step, a tomographic volume is reconstructed from the simulated radiographs using software available in a commercial XCT instrument assuming ideal alignment, i.e., assuming no geometry errors. There are about $1275 \times 1275 \times 1275$ voxels in the reconstructed volume, where each voxel is a cube of side $0.0432 \mathrm{~mm}$. The resulting voxel model is converted to a surface model by applying a gray-value threshold in VGSTUDIO MAX 3.0. Surface sampling converts the surface model to a cloud of points, where each point is defined by its three-dimensional coordinate location in the global coordinate frame. The point cloud is imported into MATLAB, and a least-squares sphere is fit to the surface coordinates of each of the 125 spheres; center coordinates, center-to-center distance errors, and form error are determined from the set of fit spheres. For the radiograph-based method, the process of generating the radiographs, reconstruction, surface extraction, sampling, and fitting is time-consuming and laborintensive, requiring several hours to complete for a single set of geometry errors.

We note that the final surface point cloud obtained is strongly dependent on the choice of gray-value threshold. The often-used ISO-50 threshold [14] does not necessarily capture the entire surface in the presence of large geometry errors; we therefore manually selected a threshold to enclose as much of the reconstructed sphere as possible.

\subsection{Single-Point Ray Tracing Method}

This method is based on the assumption that dimensional errors due to instrument geometry errors are approximately constant within small regions of the measurement volume. This assumption allows us to estimate the magnitude and direction of the errors at points located on the surface of the sphere using only the dimensional errors at the center of the sphere. Therefore, we do not forward project the entire sphere on

${ }^{2}$ Certain commercial equipment, instruments, or materials are identified in this paper to foster understanding. Such identification does not imply recommendation or endorsement by the National Institute of Standards and Technology, nor does it imply that the materials or equipment identified are necessarily the best available for the purpose. 
to the detector; instead, we only forward project the center of the sphere in the presence of individual geometry errors. The detector coordinate system is considered to be continuous; i.e., the coordinates of projected sphere centers on the plane of the detector are not limited to integer pixel values. Kumar et al. [9] also did not project the entire sphere; they discretized the spheres as a $3 \times 3 \times 3$ matrix of voxels near the sphere center. However, since their method relies on reconstruction, it is still a computationally intensive process. Further, their technique does not provide an estimate of the sphere form error. Ferrucci et al. [10] determined errors in the sphere center position and the form of the spheres, yet their method also relies on tomographic reconstruction.

The forward-projection concept is shown in Fig. 3(a). Consider a single sphere located in the measurement volume, with its center represented by point $M$ in the figure. For simplicity, in Fig. 3 and throughout this subsection, we consider the reference object as stationary, and the source-detector pair rotate about the rotation axis. Three rays pass through the sphere center $M$ and project it onto the detector. Ray 1 corresponds to the source-detector pair at an index angle $\theta$ of zero degrees. Rays 2 and 3 correspond to the source-detector pair at subsequent index angles. Figures 3(a) and (b) show that the projection on the detector of the sphere center traces an elliptical path. The locus of projected sphere centers from a full $360^{\circ}$ rotation of the source-detector pair corresponds to the radiographs generated in the radiograph-based method and is the end result of the forward-projection process. We generated sphere center trajectories on the detector in the presence of each individual instrument geometry error and used these trajectories in the subsequent back-projection step (described next) to determine the sphere center location, sphere center-tocenter distance errors, and sphere form errors.

Because no radiographs are generated during forward projection, no reconstruction is performed during back projection. Instead, back projection here involves determining the sphere center location by constructing lines connecting the source with each of the projected sphere center points on the detector. In an aligned instrument, these lines intersect at a unique point, which is coincident with the actual sphere center; see Fig. 3(b). In the presence of geometry errors, the set of lines connecting the source and the projected sphere center points on the detector do not necessarily intersect at a common point; in fact, it is possible that these lines do not intersect at all; e.g., see Fig. 3(c). In our method, the back-projection step therefore involves determining the location of the sphere center by minimizing the sum of squares of the shortest distance to each of the lines; see Fig. 3(d).

While the residuals from the above minimization can provide an indication of the sphere form, we can obtain a better visualization and quantification by constructing a circle, the diameter of which is the same as the sphere diameter, on each of the lines. The plane of the circle is perpendicular to the line on which it is constructed, and the circle center is located at the point on the line that is closest to the least-squares center determined in the previous step. For the simulation, the circles are each represented by a set of equally spaced points. A single back-projected simulated sphere surface is generated from the points pertaining to the set of constructed circles using in-built functions in MATLAB. Interior points are removed, and only points belonging to the outer surface are kept. We then fit a least-squares sphere to this point cloud to quantify and visualize form errors. We considered 360 projections with 120 points on each circle.

While the concept applied in this method is not strictly based on mathematical theorems, it has been tested for all cases of geometry errors considered in this paper. The merit of this technique lies in the fact that it can quickly provide an estimate of sphere center-to-center distance error and sphere form error for any point in the measurement volume and for any rotation axis and detector position; these results can then be confirmed using the radiograph-based method, if needed. While the single-point ray tracing method can be suitably modified to handle cases when spheres overlap, the spheres in the reference object discussed in Sec. 2.1 do not overlap. 


\section{Journal of Research of the National Institute of Standards and Technology}

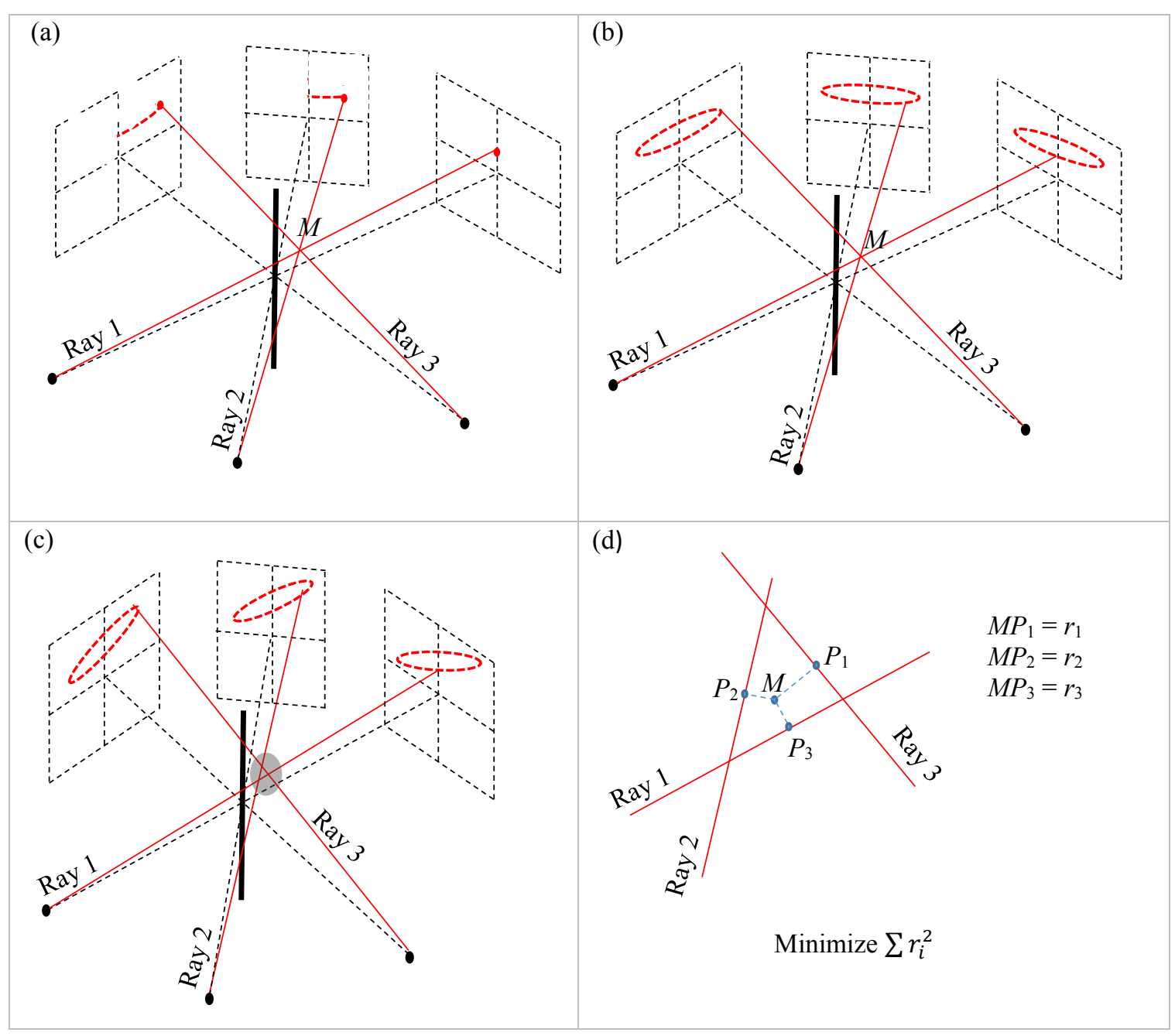

Fig. 3. (a) Ray 1 corresponds to the projection of sphere center $M$ onto the detector for the source-detector pair at an index angle $\theta$ of zero degrees. Rays 2 and 3 correspond to the projection of sphere center $M$ onto the detector for subsequent index angles. The end result of this forward-projection process is the locus of the projected points on the detector corresponding to the projection of the sphere center $M$ from the different index angles. (b) In the ideal case, three rays connecting the source to points on the detector intersect at a single point $M$, which is the location of the sphere center. (c) In the presence of geometry errors, the rays do not intersect at a common point; the region near the theoretical intersection point is shown by a shaded circle. (d) Close-up of the shaded region, where point $M$ is determined by minimizing the sum of squared distances to each ray. Point $P_{i}$ represents the point on Ray $i$ that is closest to center $M$ determined by least-squares minimization.

\subsection{Sensitivity Calculation}

The effect of geometry errors on sphere center-to-center distance error and sphere form error is determined as follows. After the sphere centers are determined, the center-to-center distance between all pairs of spheres in the measurement volume is calculated. The form error of each sphere is computed and plotted for visualization. The sensitivity coefficients, defined as the ratio of the magnitude of the distance error to the magnitude of the geometry error, and the ratio of the magnitude of the form error to the magnitude of the geometry error, are calculated. For each geometry error parameter, the distance error sensitivity value corresponding to the pair of spheres that produced the largest distance error is noted. Likewise, the form error sensitivity corresponding to the sphere that produced the largest form error is noted. The location of the spheres corresponding to these maximum sensitivity values for each considered error source is the primary target of this research. These locations will be submitted to committees within 
ASME and ISO for consideration in documentary standards for performance evaluation of XCT instruments. We note that there could be correlation between error sources; however, the scope of this study is to understand the effects of error sources independently.

\section{Error Sources Associated with the Detector}

The location and orientation of the detector are required quantities during the reconstruction process. The detector location is determined by three parameters, the $(X, Y, Z)$ coordinates of point $D$ in the global coordinate system. The detector orientation is determined by three rotation angles, each about an axis parallel to the $X, Y$, and $Z$ axes, and passing through point $D$. These quantities are typically determined by geometrical calibration of the instrument. In this section, we quantify the effects of unknown/undetermined/uncorrected errors in the detector location and orientation on the sphere center-tocenter distance error and sphere form error. The detector geometry errors are shown in Fig. 4.

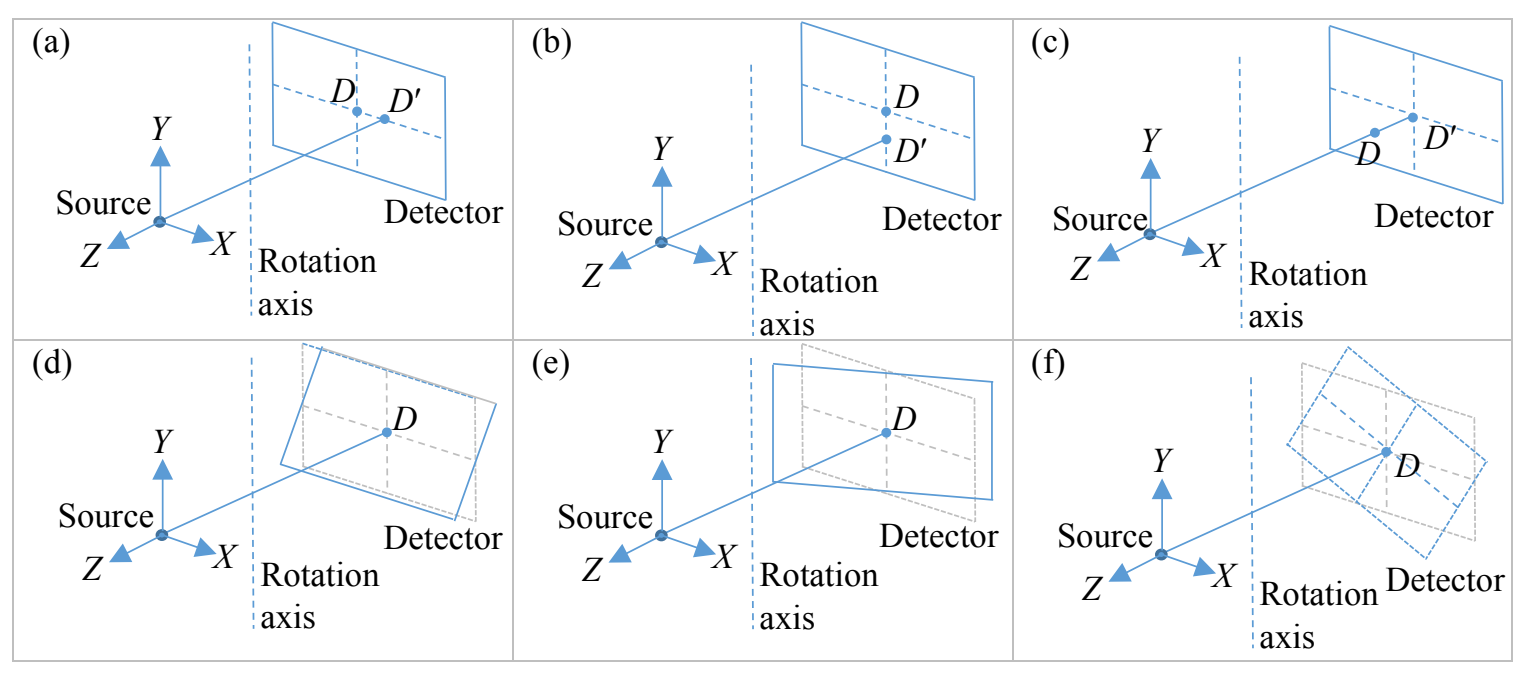

Fig. 4. Detector geometry errors are differences between assumed (by reconstruction algorithm) and actual location and orientation of the detector. (a) Detector location error in a direction parallel to the $X$ axis. (b) Detector location error in a direction parallel to the $Y$ axis. (c) Detector location error along the $Z$ axis. (d) Detector rotation error about an axis parallel to the $X$ axis. (e) Detector rotation error about an axis parallel to the $Y$ axis. (f) Detector rotation error about the $Z$ axis.

Note that the sensitivity values reported in this section are for nominal rotation axis and detector distances of $400 \mathrm{~mm}$ and $1177 \mathrm{~mm}$, respectively. The following sign convention is used for parameterizing geometry errors. For a perfectly aligned system, the true value for the coordinate of point $D$ in the global coordinate system is $(0,0,-1177) \mathrm{mm}$. Let the true value for the coordinate of point $D$ in the detector coordinate system be $(u, v)$. The true values of the detector orientation, i.e., rotation angles about the $X, Y$, and $Z$ axes, respectively, are $(0,0,0)^{\circ}$. These values are used in the back-projection process. Any deviation of the detector position and orientation from these values in the forward-projection process are considered errors. Thus, if the forward-projection step is performed with an actual detector position $D=(u+0.1, v) \mathrm{mm}$ in the detector coordinate system, the error in the $U$ coordinate of $D$ is $+0.1 \mathrm{~mm}$. If the forward-projection step is performed with actual detector orientation of $(0,0,-0.2)^{\circ}$, the error in the detector rotation angle about the $Z$ axis is $-0.2^{\circ}$. Note that rotation angles follow the right-hand rule; thus, a clockwise rotation of the detector about the $Z$ axis when viewing the detector from the source is considered a positive rotation about the $Z$ axis.

In the following sections, the magnitude of the geometry errors we considered was intentionally large and determined by trial and error to provide easily observable center-to-center distance errors or sphere form errors. Further, for any given geometry error parameter, we performed (but do not report here) 
simulations for different magnitudes of the geometry error to ensure that the center-to-center distance error and sphere form error do in fact have a linear relationship.

\subsection{Detector $X$ Location Error}

Description: This parameter describes an error in the location of point $D$ along an axis parallel to the $X$ axis, passing through point $D$.

Distance error: This error source does not result in sphere center-to-center distance errors, even for a large $X$ location error of 10 pixels $(1.27 \mathrm{~mm})$.

Form error: The form error is approximately $0.5 \mathrm{~mm}$ for an $X$ location error of 10 pixels $(1.27 \mathrm{~mm})$; therefore, the sensitivity is $0.5 / 1.27=0.4 \mathrm{~mm} / \mathrm{mm}$. This sensitivity coefficient can be thought of as dimensionless or, as shown here, in units of "mm per mm." The form error is identical for all spheres, and the spheres appear spheroidal with the short axis oriented along the $Y$ direction. Further, equivalent detector $X$ location errors in positive and negative directions result in the same form error. The form error for one of the spheres is shown in Fig. 5 on a sphere of $2 \mathrm{~mm}$ nominal diameter; form errors are color-coded, and the scale is in millimeters, for easier visualization.

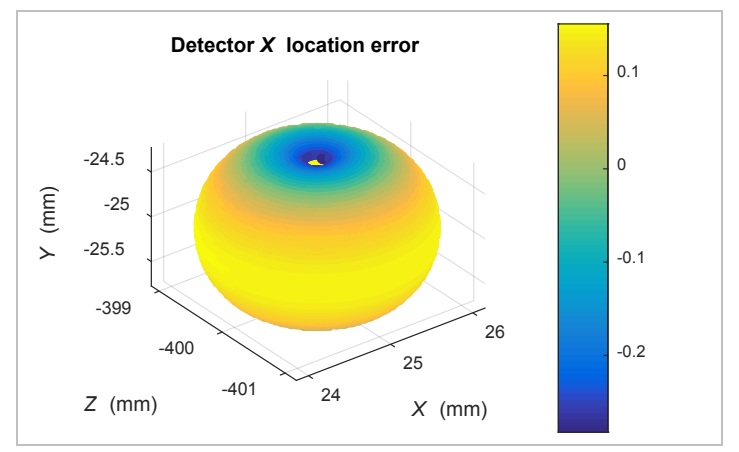

Fig. 5. Form error of the sphere located at $(25,-25,-400) \mathrm{mm}$ obtained using the single-point ray tracing method for a detector location error along the $X$ direction. The source (not shown here) is located at $(0,0,0)$, while the center of the detector (also not shown) is located at $(1.27,0,-1177) \mathrm{mm}$.

\subsection{Detector $Y$ Location Error}

Description: This parameter describes an error in the location of point $D$ along an axis parallel to the $Y$ axis and passing through point $D$.

Distance error: This error source does not result in sphere center-to-center distance errors, even for large $Y$ location error of 10 pixels $(1.27 \mathrm{~mm})$.

Form error: For spheres located on the rotation axis, this error source does not result in any significant form error. For a detector $Y$ location error of 10 pixels $(1.27 \mathrm{~mm})$, spheres located in the inner circle (25 $\mathrm{mm}$ diameter) of the reference object exhibit a form error of approximately $0.015 \mathrm{~mm}$, while the spheres located on the outer circle ( $50 \mathrm{~mm}$ diameter) exhibit a form error of approximately $0.03 \mathrm{~mm}$. The form error of the spheres has four peaks, and their orientation depends on the location of the spheres within the measurement volume. The form errors for two spheres are shown in Fig. 6. The maximum sensitivity is $0.03 / 1.27=0.02 \mathrm{~mm} / \mathrm{mm}$, which is substantially smaller than the sensitivity for the $X$ location error, implying that larger $Y$ location errors can be tolerated without significantly affecting measurements. The form error is the same for detector location errors along both the positive and negative $Y$ directions. 


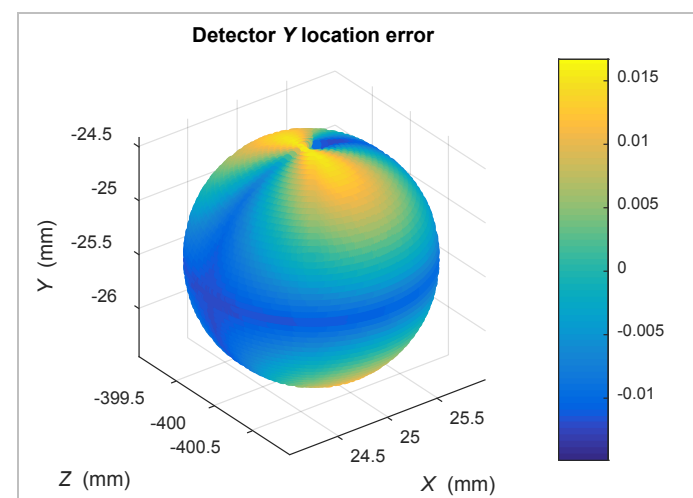

(a)

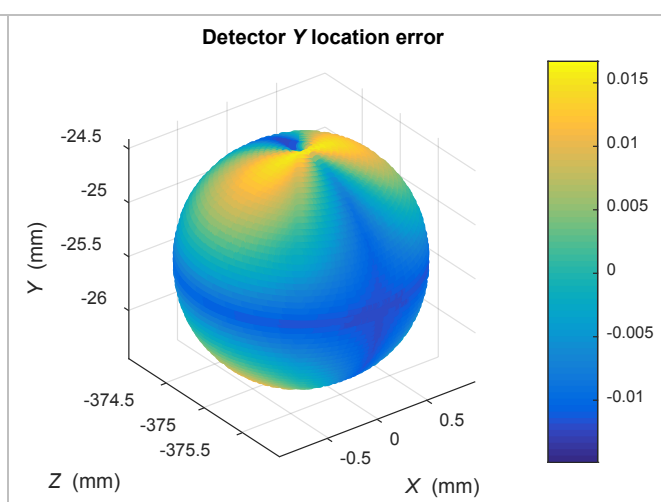

(b)

Fig. 6. Form error obtained using the single-point ray tracing method for detector location error along $Y$, shown for (a) the sphere located at $(25,-25,-400) \mathrm{mm}$ and (b) the sphere located at $(0,-25,-375) \mathrm{mm}$.

\subsection{Detector $Z$ Location Error}

Description: This parameter describes an error in the location of point $D$ along the $Z$ axis in the global coordinate system.

Distance error: Figure 7(a) shows the lines that are most sensitive to this error source. These are lines that connect spheres farthest apart from each other and passing through the center of the measurement volume $P$. We refer to such lines as the body diagonals in this paper. The distance error for these lines is approximately $-0.006 \mathrm{~mm}$ for $+0.1 \mathrm{~mm}$ error in the detector's $Z$ location and approximately $+0.006 \mathrm{~mm}$ for $-0.1 \mathrm{~mm}$ error in the detector's $Z$ location. Thus, the magnitude of the distance error sensitivity is 0.06 $\mathrm{mm} / \mathrm{mm}$.

Form error: This error source does not result in significant form error in the spheres.

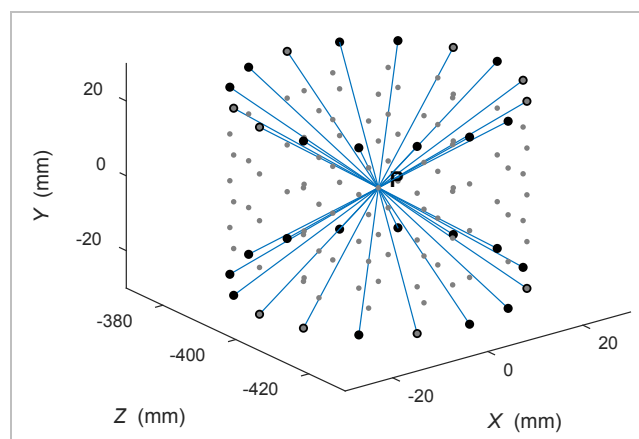

(a)

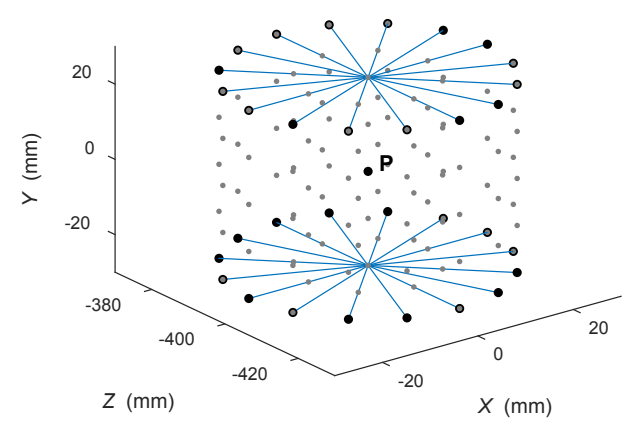

(b)

Fig. 7. (a) Sensitive lines to detect a $Z$ location error of the detector (body diagonals). (b) Sensitive lines to detect error in the rotation angle about the $X$ axis (face diagonals).

\subsection{Error in Detector Rotation Angle About the $X$ Axis}

Description: This parameter describes the error in the detector rotation angle about an axis parallel to the $X$ axis, passing through point $D$.

Distance error: Figure 7(b) shows the lines that are most sensitive to this error source. These are lines that connect spheres farthest apart from each other in a given horizontal $(X Z)$ plane and passing through the axis of rotation. We refer to such lines as the face diagonals. The face diagonals in the lowest and highest horizontal planes are most sensitive to this error source. The magnitude of the distance error for these lines 
is approximately $0.01 \mathrm{~mm}$ for $0.2^{\circ}$ of rotation about the $X$ axis; i.e., distance error sensitivity is $0.05 \mathrm{~mm} /{ }^{\circ}$. When the sign of the rotation angle error is positive, the distance errors in the bottom plane are positive, and the distance errors in the top plane are negative. When the sign of the rotation angle error is negative, the signs of the errors in the two planes reverse.

Form error: This error source does not result in any significant form error in the spheres.

\subsection{Error in Detector Rotation Angle About the $Y$ Axis}

Description: This parameter describes the error in the detector rotation angle about an axis parallel to the $Y$ axis, passing through point $D$.

Distance error: The sensitive lines are the face diagonals that pass through the rotation axis, i.e., such as those shown in Fig. 7(b), but they are located in any horizontal plane. The magnitude of the distance error is approximately $0.007 \mathrm{~mm}$ for $1^{\circ}$ of rotation about the $Y$ axis; i.e., the distance error sensitivity is $0.007 \mathrm{~mm} /{ }^{\circ}$. Thus, larger $Y$ rotation errors can be tolerated, as compared to $X$ rotation errors, without significantly affecting measurement accuracy. The errors are always positive regardless of the direction of rotation of the detector.

Form error: For spheres located on the rotation axis, this error source does not result in any significant form error. For $1^{\circ}$ of detector rotation about the $Y$ axis, spheres located in the inner circle ( $25 \mathrm{~mm}$ diameter) exhibit a form error of approximately $0.015 \mathrm{~mm}$, while the spheres located on the outer circle $(50 \mathrm{~mm}$ diameter) exhibit a form error of approximately $0.04 \mathrm{~mm}$, giving a maximum form error sensitivity of 0.04 $\mathrm{mm} /{ }^{\circ}$. There are two peaks to the form error (on diametrically opposite ends), and the orientation depends on the location of the spheres in the measurement volume. The form errors for two spheres are shown in Fig. 8. The form error does not depend on the direction of rotation of the detector.

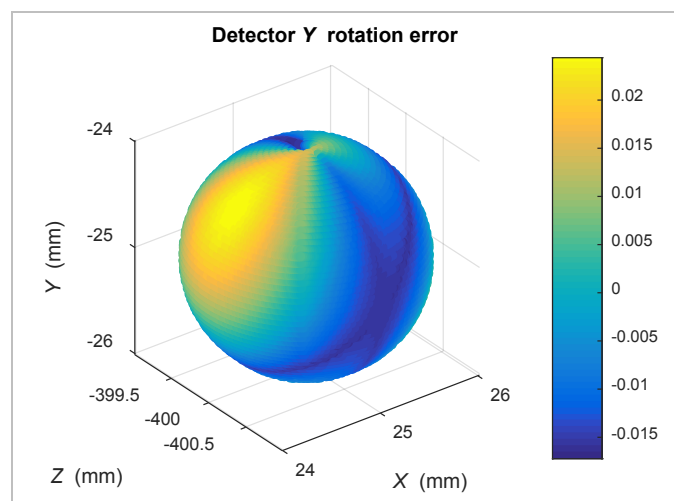

(a)

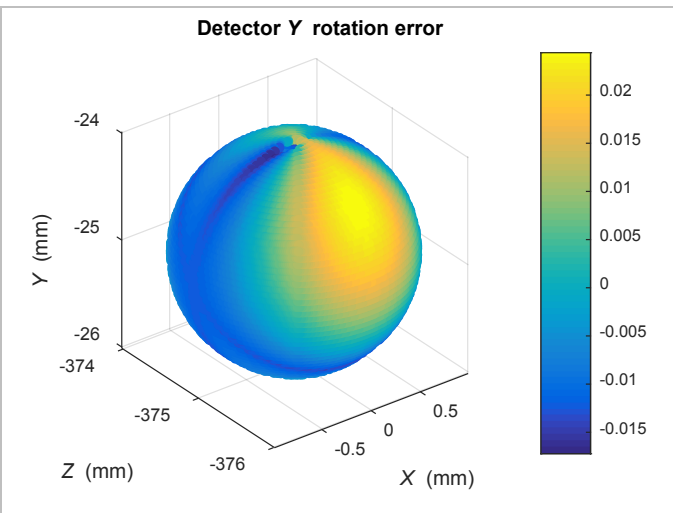

(b)

Fig. 8. Form error obtained using the single-point ray tracing method for detector rotation error about the $Y$ axis, shown for (a) the sphere located at $(25,-25,-400) \mathrm{mm}$ and (b) the sphere located at $(0,-25,-375) \mathrm{mm}$.

\subsection{Error in Detector Rotation Angle About the $Z$ Axis}

Description: This parameter describes the error in the detector rotation angle about the $Z$ axis, i.e., inplane rotation.

Distance error: This error source does not result in sphere center-to-center distance errors, even for large rotation error of $0.2^{\circ}$ about the $Z$ axis.

Form error: The form error depends on the location of the sphere. There is no form error exhibited on the sphere located at the center of the measurement volume (point $P$ in Fig. 1). The form error increases radially outwards in any horizontal plane and also increases with increasing distance from the $Y=0$ plane. 
For $0.2^{\circ}$ of rotation of the detector about the $\mathrm{Z}$ axis, the spheres located on the outer circle $(50 \mathrm{~mm}$ diameter) at the lowest and highest planes exhibit a form error of approximately $0.15 \mathrm{~mm}$. The spheres are squeezed along the line joining their center and the center of the measurement volume. The form errors for two spheres are shown in Fig. 9. The maximum form error sensitivity is approximately 0.15/0.2 $=0.75$ $\mathrm{mm} /{ }^{\circ}$. The magnitude and shape of the form error do not depend on the direction of rotation of the detector.

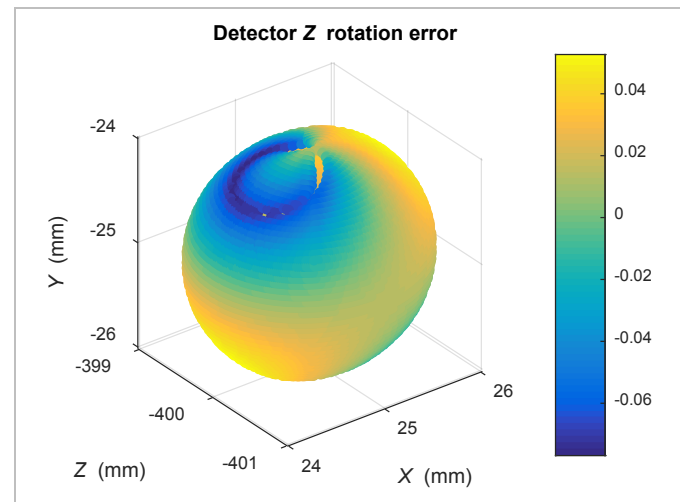

(a)

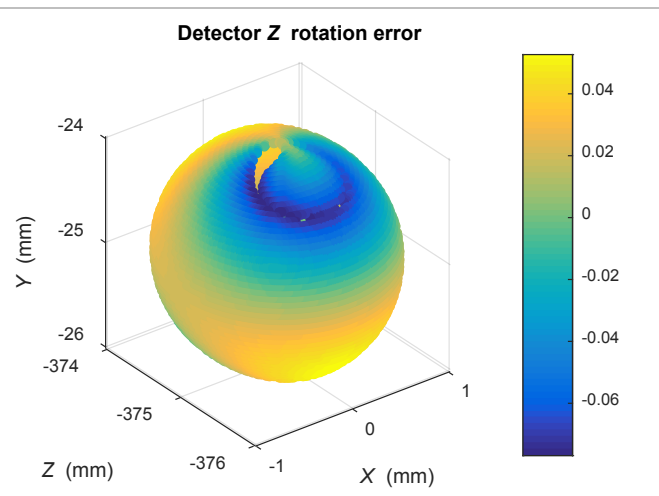

(b)

Fig. 9. Form error obtained using the single-point ray tracing method for detector rotation error about the $Z$ axis, shown for (a) sphere located at $(25,-25,-400) \mathrm{mm}$ and (b) sphere located at $(0,-25,-375) \mathrm{mm}$.

\subsection{Sensitivity Matrix for Detector Errors}

We discussed the sensitivity of distance and form error to different detector geometry errors in the previous subsections. In Table 1, we summarize these sensitivities in the form of a sensitivity matrix using the eight spheres $(A-H)$ shown in Fig. 10. Spheres $A, B, C$, and $D$ are located on the bottom plane $(Y=-25$ $\mathrm{mm})$ and on a circle of $50 \mathrm{~mm}$ diameter. Spheres $E, F, G$, and $H$ are located on the top plane $(Y=25 \mathrm{~mm})$ and on a circle of $50 \mathrm{~mm}$ diameter. The spheres create four body diagonals $(A G, C E, D F, B H)$ and four face diagonals $(A C, B D, F H, E G)$.

At the initial angular position of the reference object (index angle of $0^{\circ}$ ), spheres $A, C, E$, and $G$ form a plane parallel to the detector, while spheres $B, D, F$, and $H$ form a plane perpendicular to the detector. Dimensional measurements of these eight spheres are the most sensitive to the different detector geometry errors discussed earlier.

Here, we summarize the sensitivity observations made in the previous sections as they pertain to the sensitive sphere locations $(A-H)$ in the reference object. Table 1 shows that distance errors are most sensitive to detector $Z$ location error and detector rotation about the $X$ axis. Distance errors are moderately sensitive to detector rotation about the $Y$ axis. Sphere form errors are sensitive to detector $X$ location error and detector rotation about the $Z$ axis. Form errors are moderately sensitive to detector $Y$ location error and detector rotation about the $Y$ axis. 


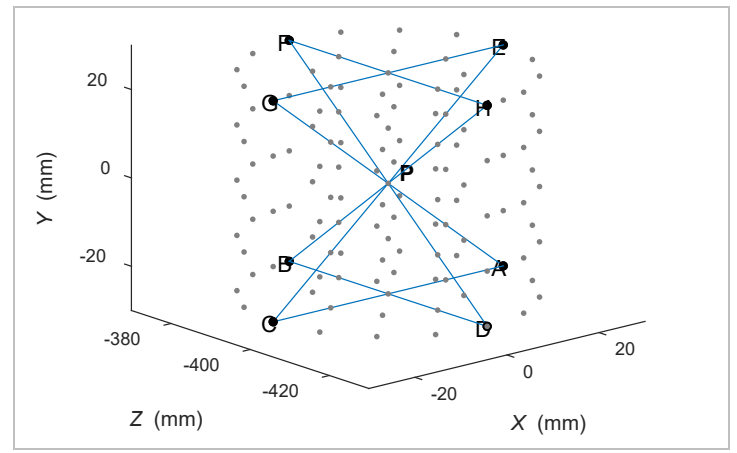

Fig. 10. Eight out of the 125 spheres, forming four face diagonals and four body diagonals, can be used to capture all detector geometry errors.

Table 1. Sensitivity matrix for detector errors based on the eight spheres shown in Fig. 10.

\begin{tabular}{|c|c|c|c|c|c|}
\hline \multirow[t]{2}{*}{ Source } & \multicolumn{2}{|c|}{ Distance error } & \multicolumn{2}{|c|}{ Form error } & \multirow[t]{2}{*}{ Description } \\
\hline & Lines & Sensitivity $^{\mathrm{a}}$ & Spheres $^{\mathrm{b}}$ & Sensitivity ${ }^{\mathrm{a}}$ & \\
\hline$X$ location & None & 0 & All & $\begin{array}{c}0.4 \\
\mathrm{~mm} / \mathrm{mm}\end{array}$ & Form error: Spheres are elongated in the $X Z$ plane \\
\hline$Y$ location & None & 0 & All & $\begin{array}{c}0.02 \\
\mathrm{~mm} / \mathrm{mm}\end{array}$ & $\begin{array}{l}\text { Form error: Four lobes, spheres are elongated along the } Y \\
\text { direction, orientation of lobes depends on position of } \\
\text { sphere }\end{array}$ \\
\hline$Z$ location & $\begin{array}{l}A G, C E \\
D F, B H\end{array}$ & $\begin{array}{c}0.06 \\
\mathrm{~mm} / \mathrm{mm}\end{array}$ & None & 0 & Distance error: Body diagonals passing through $P$ \\
\hline $\begin{array}{l}\text { Rotation } \\
\text { about } X\end{array}$ & $\begin{array}{l}A C, B D \\
F H, E G\end{array}$ & $0.05 \mathrm{~mm} /{ }^{\circ}$ & None & 0 & $\begin{array}{l}\text { Distance error: Face diagonals that pass through the } \\
\text { rotation axis in the lowest and highest horizontal planes }\end{array}$ \\
\hline $\begin{array}{l}\text { Rotation } \\
\text { about } Y\end{array}$ & $\begin{array}{l}A C, B D, \\
F H, E G\end{array}$ & $0.007 \mathrm{~mm} /{ }^{\circ}$ & All & $0.04 \mathrm{~mm} /{ }^{\circ}$ & $\begin{array}{l}\text { Distance error: Sensitive to face diagonals in any } \\
\text { horizontal plane } \\
\text { Form error: Spheres are elongated along the line joining } \\
\text { their center and the center of the measurement volume } P\end{array}$ \\
\hline $\begin{array}{l}\text { Rotation } \\
\text { about } Z\end{array}$ & None & 0 & All & $0.75 \mathrm{~mm} /{ }^{\circ}$ & $\begin{array}{l}\text { Form error: Spheres are squeezed along the line joining } \\
\text { their center and the center of the measurement volume } P\end{array}$ \\
\hline
\end{tabular}

\section{Discussion}

\subsection{Sensitive Sphere Locations for Detector Errors}

Our recommendation for sphere center locations to detect detector geometry errors are as follows (see Fig. 10):

- Four spheres should be located at index angles of $0^{\circ}, 90^{\circ}, 180^{\circ}$, and $270^{\circ}$, radially as far away from the axis of rotation as possible and in the lowest $Y$ position possible, when the rotation stage is at the index angle of zero degrees.

- Four spheres should be located at index angles of $0^{\circ}, 90^{\circ}, 180^{\circ}$, and $270^{\circ}$, radially as far away from the axis of rotation as possible and in the highest $Y$ position possible, when the rotation stage is at the index angle of zero degrees.

- The four spheres in the lower horizontal plane and the four in the upper horizontal plane allow for the determination of four face diagonals $(A C, B D, E G, F H)$.

- These spheres also allow for the determination of four body diagonals $(A G, C E, D F, B H)$. 
- The face and body diagonals as described in the preceding two bullets are recommended lines to capture detector geometry errors.

- The form errors of the eight spheres are sensitive to some detector geometry errors as discussed in this paper. Monitoring form error of spheres located in the measurement volume, especially those located further away from $P$ along both the radial and $Y$ directions, is therefore a quick and easy test of some detector geometry errors.

\subsection{Validation of the Single-Point Ray Tracing Method}

We present some data here demonstrating the agreement between the single-point ray tracing method and the radiograph-based method. Figure 11(a) shows sphere center-to-center distance errors (for a few selected sphere pairs) for the single-point ray tracing and the radiograph-based method in the presence of rotation axis $Z$ location error, while Fig. 11(b) shows the differences between the methods. Agreement between the methods is better than $2 \mu \mathrm{m}$. Even though there is a discernible difference between the methods (albeit small), it is important to note that the radiograph-based method does not necessarily represent the ideal. The ideal setup to study the influence of geometric errors on point-to-point distances would be the radiograph approach with arbitrarily small spheres. However, the radiograph method needs spheres of reasonable size, meaning that the single-point ray tracing method may — for the purposes hereactually be superior to the radiograph-based method, at least for the center-to-center distance errors.

Figure 11(c) shows the form error for one of the spheres in the presence of error in the detector rotation angle about the $Z$ axis using the radiograph-based method, while Fig. 11(d) shows the form error for the same sphere using the single-point ray tracing method. We note that the radiograph-based method resulted in a reconstructed form error on the order of $0.03 \mathrm{~mm}$ for a perfect sphere, i.e., a sphere with no form error. This nonzero form error is likely due to the method adopted to generate the radiographs in MATLAB, and the interpolation performed during reconstruction and surface determination. Thus, the agreement in the form error $(0.14 \mathrm{~mm}$ for the radiograph-based method versus $0.13 \mathrm{~mm}$ for the single-point ray tracing method) between the two methods in Fig. 11, and others not presented, to within a few tens of micrometers indicates that the single-point ray tracing method is in fact capable of modeling and predicting the form error in the presence of instrument geometry errors.

We provide another example in Figs. 11(e) and (f). Figure 11(e) shows the form error for one of the spheres in the presence of detector $X$ location error using the radiograph-based method, while Fig. 11(f) shows the form error for the same sphere using the single-point ray tracing method. The agreement in the form error $(0.5 \mathrm{~mm}$ for the radiograph-based method versus $0.44 \mathrm{~mm}$ for the single-point ray tracing method) is again within a few tens of micrometers. We performed such comparisons for all error sources described in this paper. 


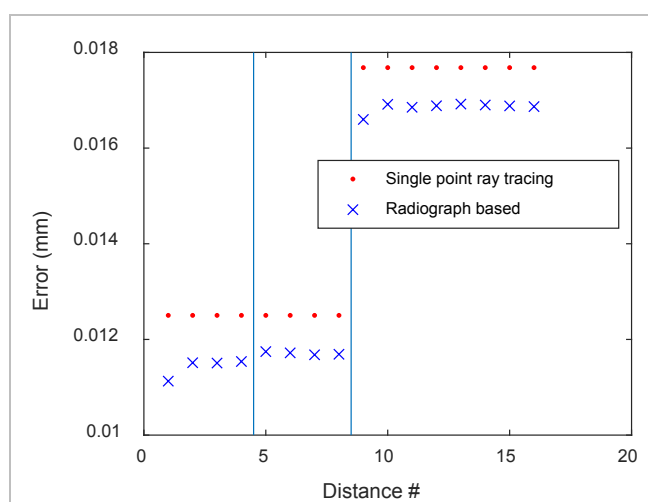

(a)

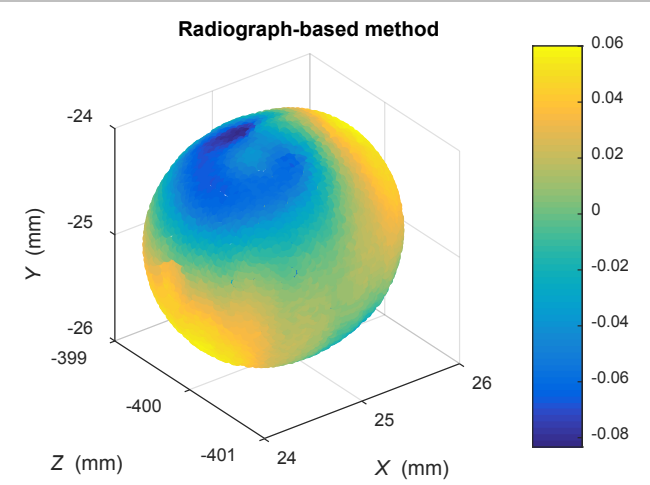

(c)

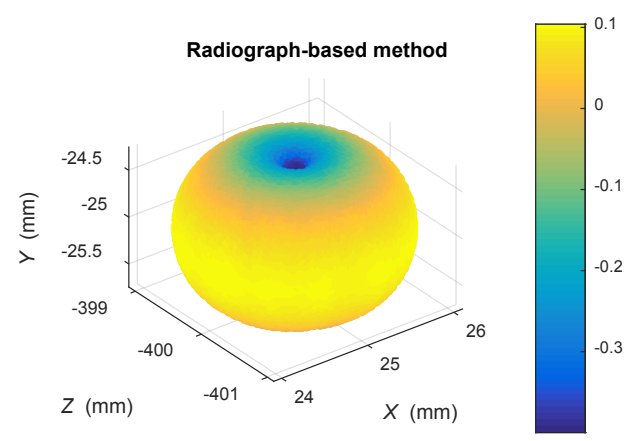

(e)

Fig. 11. (a) Distance error in presence of rotation axis $Z$ location error for a few selected distances. Distances 1 through 4 are the face diagonals for the lowest $Y$ position, distances 5 through 8 are the face diagonals for the highest $Y$ position, and distances 9 through 16 are the body diagonals. (b) Differences in the errors between the methods. (c) Form error for a sphere in the presence of error in the detector rotation angle about the $Z$ axis using the radiograph-based method. (d) Form error for the same sphere using the single-point ray tracing method. (e) Form error for a sphere in the presence of detector $X$ location error using the radiograph-based method. (d) Form error for the same sphere using the single-point ray tracing method.

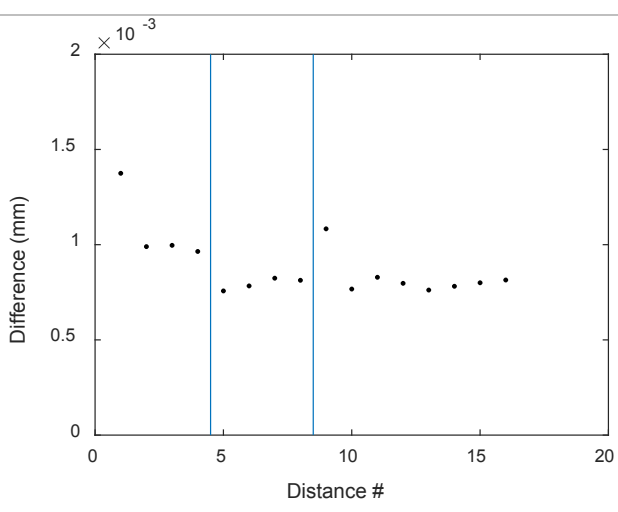

(b)

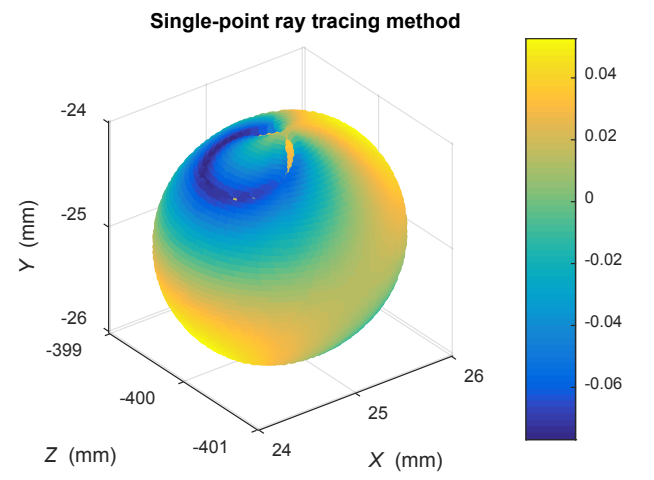

(d)

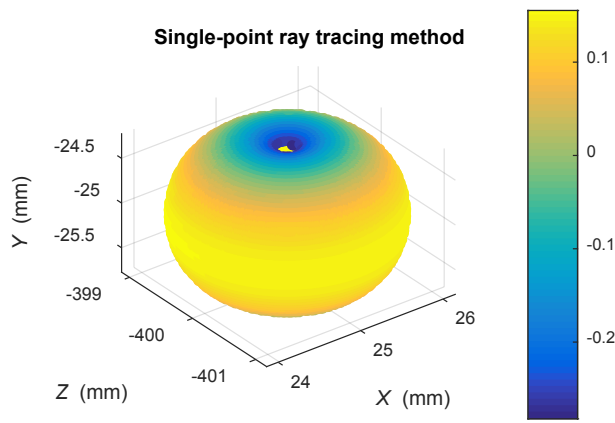

(f) 


\section{Conclusions}

For the nominal rotation axis and detector distances of $400 \mathrm{~mm}$ and $1177 \mathrm{~mm}$, respectively, we described the effect of XCT detector geometry errors on sphere center-to-center distance error and sphere form error for spheres located in the measurement volume. We performed our simulations using two different approaches, the radiograph-based method and the single-point ray tracing method.

The radiograph-based method involves generating radiographs of the spheres in the presence of geometry errors, reconstructing a voxel model from the radiographs assuming ideal geometry, and performing surface determination and fitting to determine sphere centers, followed by calculation of sphere center-to-center distance error and sphere form error. This method is labor-intensive because it requires the use of multiple pieces of software and takes several hours on a personal computer for each set of radiographs.

We therefore developed a new single-point ray tracing method, where sphere centers are forward projected onto the detector in the presence of geometry errors, and the rays are subsequently back-projected assuming ideal geometry to determine sphere center locations using a least-squares approach. This method does not involve any reconstruction and therefore only takes a few minutes on a personal computer.

Using the single-point ray tracing method (and validated using the radiograph-based method), we quantified the sensitivity of the distance and form error to each of the detector geometry error parameters considered in this paper. Based on the analysis, we made recommendations on positions of spheres in the measurement volume to detect these geometry errors. In Part II, we discuss the effect of rotation stage errors on sphere center-to-center distance error and sphere form error and make recommendations on sphere center locations that are sensitive to those errors. The motivation for this work is to provide input to ongoing standards efforts within ASME and ISO so that the test procedures they specify are sensitive to all known geometry errors.

\section{Acknowledgments}

The first author is grateful to Massimiliano Ferrucci of KU Leuven for helpful discussions during the course of this work and for his careful review of the paper. The authors thank Dr. Craig Shakarji of the National Institute of Standards and Technology (NIST), Dr. Valentina Aloisi of North Star Imaging, Inc., Dr. Toshiyuki Takatsuji of the National Metrology Institute of Japan (NMIJ), and Dr. Herminso VillarragaGómez of Nikon Metrology, Inc., for their feedback.

\section{References}

[1] Kruth JP, Bartscher M, Carmignato S, Schmitt R, De Chiffre L, Weckenmann A (2011) Computed tomography for dimensional metrology. CIRP Annals 60(2):821-842. https://doi.org/10.1016/j.cirp.2011.05.006

[2] Thompson A, Maskery I, Leach RK (2016) X-ray computed tomography for additive manufacturing: A review. Measurement Science and Technology 27(7):072001. https://doi.org/10.1088/0957-0233/27/7/072001

[3] Phillips S, Krystek M, Shakarji C, Summerhays K (2009) Dimensional measurement traceability of 3D imaging data. Proceedings of the SPIE 7239:72390E. https://doi.org/10.1117/12.816498

[4] American Society of Mechanical Engineers (2006) ASME B89.7.5-2006-Metrological traceability of dimensional measurements to the SI unit of length (ASME, New York).

[5] The Association of German Engineers (2011) VDI/VDE 2617-13-Guideline for the application of DIN EN ISO 10360 for coordinate measuring machines with CT sensors (Association of German Engineers, Düsseldorf, Germany).

[6] Carmignato S, Dewulf W, Leach R (Eds) (2018) Industrial X-ray Computed Tomography (Springer, Münich, Germany).

[7] Welkenhuyzen F, Kiekens K, Pierlet M, Dewulf W, Bleys P, Kruth JP, Voet A (2009) Industrial computer tomography for dimensional metrology: Overview of influence factors and improvement strategies. Proceedings of the 4th International Conference on Optical Measurement Techniques for Structures and Systems: OPTIMESS2009, pp 401-410.

[8] Ferrucci M, Leach RK, Giusca C, Carmignato S, Dewulf W (2015) Towards geometrical calibration of X-ray computed tomography systems - A review. Measurement Science and Technology 26(9):092003. https://doi.org/10.1088/09570233/26/9/092003

[9] Kumar J, Attridge A, Wood PKC, Williams MA (2011) Analysis of the effect of cone-beam geometry and test object configuration on the measurement accuracy of a computed tomography scanner used for dimensional measurement. Measurement Science and Technology 22(3):035105. https://doi.org/10.1088/0957-0233/22/3/035105 
[10] Ferrucci M, Ametova E, Carmignato S, Dewulf W (2016) Evaluating the effects of detector angular misalignments on simulated computed tomography data. Precision Engineering 45:230-241. https://doi.org/10.1016/j.precisioneng.2016.03.001

[11] Ametova E, Ferrucci M, Chilingaryan S, Dewulf W (2018) A computationally inexpensive model for estimating dimensional measurement uncertainty due to X-ray computed tomography instrument misalignments. Measurement Science and Technology 29(6):065007. https://doi.org/10.1088/1361-6501/aab1a1

[12] Aloisi V, Carmignato S, Schlecht J, Ferley E (2017) Investigation on the effects of X-ray CT system geometrical misalignments on dimensional measurement errors. Proceedings of the 7th Conference on Industrial Computed Tomography (Leuven, Belgium).

[13] Ferrucci M, Heřmánek P, Ametova E, Carmignato S, Dewulf W (2018) Measurement of the X-ray computed tomography instrument geometry by minimization of reprojection errors-Implementation on simulated data. Precision Engineering 54:720. https://doi.org/10.1016/j.precisioneng.2018.03.012

[14] Lifton JJ, Malcolm AA, McBride JW (2015) On the uncertainty of surface determination in X-ray computed tomography for dimensional metrology. Measurement Science and Technology 26(3):035003. https://doi.org/10.1088/0957-0233/26/3/035003

About the authors: Bala Muralikrishnan and Meghan Shilling are mechanical engineers in the Sensor Science Division at NIST. Steve Phillips was a physicist in the Sensor Science Division at NIST. Wei Ren is a computer scientist in the Sensor Science Division at NIST. Vincent Lee is a mechanical engineer in the Sensor Science Division at NIST. Felix Kim is a mechanical engineer in the Intelligent Systems Division at NIST. The National Institute of Standards and Technology is an agency of the U.S. Department of Commerce. 